\title{
Probing Vortex Density Fluctuations in Superfluid Turbulence
}

\author{
P.-E. Roche ${ }^{1}$, B. Chabaud ${ }^{1}$, O. Français ${ }^{2}$, L. Rousseau ${ }^{2}$, and H. Willaime ${ }^{3}$ \\ 1 Institut Néel - CNRS, BP166, 38042 Grenoble Cedex 9, France \\ 2 Groupe ESIEE, BP99 - 93162 Noisy le Grand Cedex, France \\ 3 ESPCI, 10 rue Vauquelin, 75231 Paris Cedex 05, France
}

\section{Introduction}

The flow dynamics of a superfluid can be described by a viscousless tangle of quantized vortex lines $[1,2,3,4]$. In spite of these unusual mechanical properties, strong similarities have been reported between superfluid (or "quantum") turbulence and its Navier-Stockes counterpart. Most of them were detected by macroscopic sensors probing the largest scales (integral length) of the flow. At smaller scales, the zero-viscosity and quantization is expected to ruin this similarity between the two turbulences but probing the inertial range is already an experimental challenge [5]. In a keystone experiment, Maurer and Tabeling measured local fluctuations in a bulk flow of helium with and without superfluid[6]. In both case, they found a Kolmogorov-like spectrum for velocity in the inertial range, which has been confirmed since by numerical simulations $[7,8,9]$.

Recently, we micro-machined and operated a local probe of the vortex line density fluctuations (projected on a plane). It provides a second direct experimental characterization of the inertial range of superfluid turbulence. The flow, the operation of this probe and the first physical results are presented in another paper[10]. On figure 1 (left), we illustrate these results with power spectra of the vortex line density. In the present paper, we present further details on micro-machining of the probe. To our knowledge, this is the first fully micro-machined sensor used in a cryogenic turbulence experiment.

\section{Flow and Probe}

\section{The flow}

The flow is confined in a cryostat and continuously powered by a centrifugal pump (see figure 1). Turbulence is probed in a $23 \mathrm{~mm}$-diameter pipe, located upstream from the pump. Velocity can span the range $0.25-1.3 \mathrm{~m} / \mathrm{s}$. The 


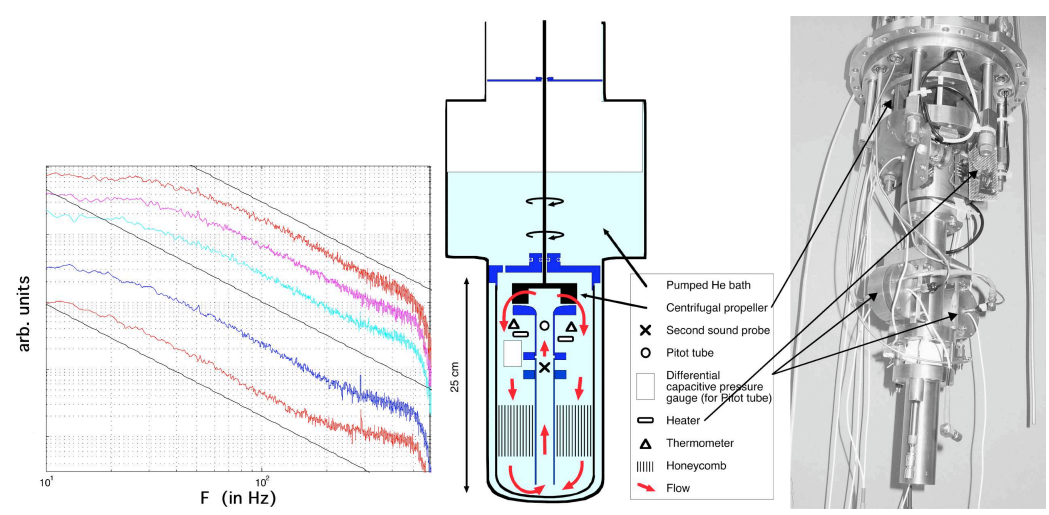

Fig. 1. (Left) Power spectrum of the projected vortex line density for a mean velocities from 0.25 (bottom curve) up to $0.78 \mathrm{~m} / \mathrm{s}$ (top curve). Saturations at high frequencies result from the noise floor and filtering. Solid lines are $-5 / 3$ power laws. (Center and Right) The closed loop of He.

measurements are performed in ${ }^{4} \mathrm{He}$ near $1.6 \mathrm{~K}$. In such conditions, $84 \%$ of helium is superfluid, the rest being a normal (viscous) fluid superimposed to the superfluid. More details are presented in [10].

\section{The probe's micro-machining}

The principle of operation of the sensor is presented in [10]. In short, it consists in an open second-sound resonator inserted across the flow. Second sound (thermal waves) is known to be attenuated by the vortex lines which are polarized in the plane of propagation[1]. Thus, a time-resolved measurement of the standing wave amplitude provides a direct measurement of the total length of the (projected) vortices within the fluid element advected through the resonator. This signal is somehow analog to an enstrophy defined using a projected vorticity.

Both mirrors of the cavity are $15 \mu \mathrm{m}$ thick silicon beams separated by a $250 \mu \mathrm{m}$ gap. The length and width of both beams is $1.5 \mathrm{~mm} \times 1 \mathrm{~mm}$ and they are facing each other with a lateral positioning within a few tenths of $\mathrm{mm}$ typically. A granular Al film is deposited over a $0.8 \mathrm{~mm}$ square area at the tip of one beam. This film is used as transition edge superconducting thermometer. Facing this thermometer, a chromium heating film is deposited on the tip of the other beam. Between both beams, a third silicon spacer sets the gap of the cavity. On both sides of it, contact pads provide electrical connections of both beams to the electronic circuit.

The micro-machining process is given in figure 2. SOI wafers (4 inches diameter) have been used with the following typical thicknesses of the 3 layers : $15 \mu \mathrm{m}, 0.5 \mu \mathrm{m}$ and $500 \mu \mathrm{m}$. Figure 3 (left and center) shows the wafer with 


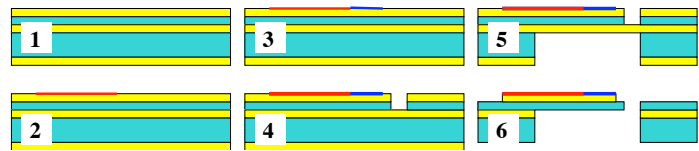

Fig. 2. Steps of the micro-machining process of one beam (the other beam is machined following a similar procedure). 1-Electrical isolation (thermal oxidation, 400 $\mathrm{nm}$ ), 2-contact pads (Cr $150 \mathrm{~nm}, \mathrm{Au} 500 \mathrm{~nm}$ ), 3-Heater (Cr, $500 \mathrm{~nm}), 4$-DRIE front side $(15 \mu \mathrm{m}), 5$-DRIE back side $(500 \mu \mathrm{m}), 6$-oxide, cleaning...

beams of various sizes. The picture on figure 3 shows the resonator after assembly.

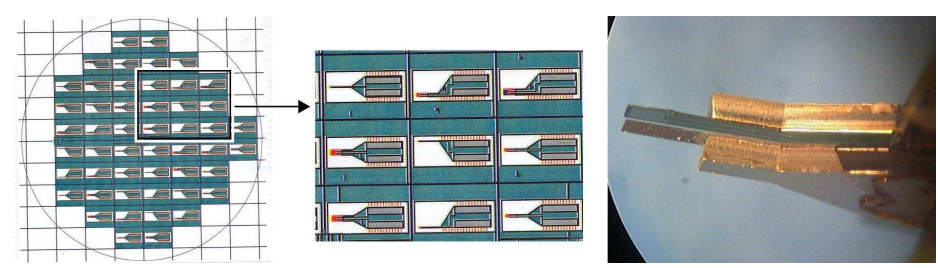

Fig. 3. (Left) Schematics of a region of the wafer, at the end of the micro-machining process. (Right) Picture of the tip of the probe after assembly.

\section{acknowledgments}

We thanks P. Tabeling, C. Lemonias, E. André, T. Fournier, B. Hébral and B. Castaing for their inputs. We acknowledge financial support from the IPMC, the Région Rhônes-Alpes and of the ANR (TSF project).

\section{References}

1. R. J. Donnelly: Quantized Vortices in Helium-II (Cambridge Studies in Low Temperature Physics, Cambridge University Press, Cambridge 1991)

2. S. K. Nemirovskii and W. Fiszdon:Rev. Modern Phys. 67, 37 (1995)

3. C.F. Barenghi et al. (Ed.): Quantized Vortex Dynamics and Superfluid Turbulence (Springer, Lecture Notes in Physics 571, 2001)

4. W. F Vinen and J. J Niemela:J. Low Temp. Phys. 128, 167 (2002)

5. W. F Vinen:J. Low Temp. Phys. 124, 101 (2001)

6. J. Maurer and P. Tabeling:Europhys. Lett. 1, 29 (1998)

7. C. Nore et al.:Phys. Fluids 9, 2644 (1997)

8. D. Kivotides et al.:Europhys. Lett. 57,845 (2002)

9. M. Kobayashi and M. Tsubota:Phys. Rev. Lett. 94, 065302 (2005)

10. to appear in Europhys. Lett (2007) 